\title{
The appropriateness of pathological reports
}

\section{Opinion}

In these last ten years, pathologist's role in multidisciplinary equipment is rapidly evolving. We can picture pathologists facing a microscope in a lab rather than a clinician with a patient. Although most patients may never even meet one, there may be no specialist more important to ensuring the most precise cancer treatment, especially regarding the knowledge on the molecular basis of cancerogenesis.

Pathologists are faced with two different kinds of relationships: one with the patient (from whom the specimen was taken) and one with the patient's doctor, either a specialist or a general practitioner.

Therefore, a pathologist's report should be intelligible to address differing significantly from a cultural and a linguistic point of view but having the same needs: discovering pathologies quickly and accurately in order to programme a well-timed therapy.

In the light of these facts, pathologists' reports should be concise (stating the final diagnosis briefly), clear (easily interpretable, though not giving up the usually necessary scientific terminology) and have a univocal interpretation (understood by both specialist and family doctors despite their different training).

In any case, the appropriateness of pathological reports cannot be done without the evolution of etiological and pathogenetical findings of illnesses, together with a complete and complex evaluation of the prognostic factors of cancers. These aspects are of paramount importance to oncologists, who must staging the illness and that may use new drugs in the target therapy, which has also been legislatively approved for more and more neoplastic diseases.

Therefore, an appropriate report should be complete. Where possible, it must include information about the biological and clinical course of the illness and all the data useful for the definition of prognostic factors.

Moreover, pathologists' reports should be precise and unequivocally specify the received and examined tissue, any technical and special stains integration into normal procedures for diagnostic reasons, and the clinical and anamnestic data which are believed helpful for pathological diagnoses.

In the light of this, pathologists organise their final report in a complex way, again with the aim of giving more complete and clearer information about the pathology in question.

In order to give the best diagnostic tool on a bioptic or surgical specimen, macroscopic observation should never be considered of minor importance as pathologists are mainly "doctors of morphology". As such, a complete pathological report cannot be done without a descriptive observation of the extracted tissue. Ancillary colouring techniques are an essential aid to the final diagnosis, but should not lead to conclusions based on their interpretation only.

The final diagnosis of reports should result from an integration of morphological data and biological information obtained from the tissue (immunophenotype, molecular biology) together with clinical, serological and anamnestic data of the patient from whom the specimen for the pathological diagnosis has been taken.
Volume 5 Issue 3 - 2018

\author{
Stefania Erra \\ Department of Surgical Pathology, Santo Spirito Hospital, Italy
}

Correspondence: Stefania Erra, Department of Surgical Pathology, Santo Spirito Hospital, Casale Monferrato, 15033, Italy, Tel +390 I 42434894, Email stefania.erra@virgilio.it

Received: May 29, 2018 | Published: June 13, 2018

Last but not least, the final pathological report has to be written in order to safeguard all surgical pathology equipment staff from legal measures in case of patients' complaints against inappropriate medical treatments.

In conclusion, pathological final diagnosis has to be draw up in a complete but concise report, including all data useful for deciding the most appropriate personalized therapy for that particular patient and for that specific pathology. In order to obtain a "gold standard" in pathological report, the figure of the pathologist must necessarily be valued above all in the context of the multidisciplinary medical team for the management of neoplastic diseases.

\section{Acknowledgements}

None.

\section{Conflict of interest}

The author declares no conflict of interest. 